\title{
Insulin signaling is required for insulin's direct and indirect action on hepatic glucose production
}

\author{
Simon J. Fisher and C. Ronald Kahn \\ Research Division, Joslin Diabetes Center; and Department of Medicine, Harvard Medical School; Boston, Massachusetts, USA
}

\begin{abstract}
We and others have suggested that insulin predominantly acts indirectly to inhibit hepatic glucose production (HGP) via suppression of gluconeogenic precursors, FFAs, and glucagon. To test that hypothesis, we performed high-dose hyperinsulinemic-euglycemic clamps using $\left[3-{ }^{3} \mathrm{H}\right]$-glucose in liver-specific insulin receptor knockout (LIRKO) mice, LIRKO mice treated with streptozotocin (LIRKO+STZ), and controls. In LIRKO mice, fasted glucose was normal, but insulin levels were elevated tenfold. STZ treatment reduced insulinemia by $60 \%$ with resulting hyperglycemia. Interestingly, basal HGP was similar in all three groups. During the clamp, HGP was suppressed by $82 \pm 17 \%$ in controls, but was not suppressed in either LIRKO or LIRKO+STZ mice. Glucose infusion and utilization were impaired $(-50 \%)$ in LIRKO and LIRKO+STZ mice versus controls. Insulin suppressed FFAs similarly in all groups $(\sim 46 \%)$. Glucagon was not significantly suppressed during the clamp. Thus, in LIRKO mice, (a) high-dose insulin fails to suppress HGP indicating that both direct and indirect effects of insulin require an intact insulin-signaling pathway in the liver; (b) primary hepatic insulin resistance leads to hyperinsulinemia and secondary extrahepatic insulin resistance; and (c) lowering insulin levels with STZ tended to improve extrahepatic insulin sensitivity but failed to reveal the previously postulated indirect role of insulin in suppressing HGP.
\end{abstract}

J. Clin. Invest. 111:463-468 (2003). doi:10.1172/JCI200316426.

\section{Introduction}

The failure of insulin to inhibit hepatic glucose production (HGP) is a cardinal feature of insulin resistance in the liver and a major factor contributing to hyperglycemia in diabetic states. Classically, the HGP-suppressing effect of insulin is thought to occur via signaling through the hepatic insulin receptor to inhibit glycogenolysis and gluconeogenesis. This classical paradigm has been challenged by in vivo studies suggesting that much of insulin's action on the liver may be indirect, and related to systemic, rather than hepatic, insulin effects (1-6). Thus, extrahepatic actions of insulin on muscle and adipose tissue to inhibit release of gluconeogenic substrates (e.g., lactate, alanine, and glycerol) and gluconeogenic energy substrates (e.g., FFAs) result in a subsequent inhibition of HGP (7-10). These mechanisms may be particularly important in the diabetic state, in which gluconeogenesis is considerably increased (11-14), and in which hyperglycemia per se has been shown to potentiate the indirect actions of

Received for publication July 15, 2002, and accepted in revised form November 19, 2002.

Address correspondence to: $\mathrm{C}$. Ronald Kahn, Joslin Diabetes Center, One Joslin Place, Room 705, Boston, Massachusetts 02215, USA. Phone: (617) 732-2635; Fax: (617) 732-2487; E-mail: c.ronald.kahn@joslin.harvard.edu.

Conflict of interest: The authors have declared that no conflict of interest exists.

Nonstandard abbreviations used: hepatic glucose production (HGP); liver-specific insulin receptor knockout (LIRKO); streptozotocin (STZ); not significant (NS). insulin (15). Furthermore, since up to $75 \%$ of basal HGP is driven by glucagon (16), insulin-induced suppression of glucagon release may be another indirect mechanism by which insulin suppresses $\operatorname{HGP}(2,3,17,18)$.

We have recently developed a unique model to investigate direct versus indirect insulin actions. Liver-specific insulin receptor knockout (LIRKO) mice have an approximately $95 \%$ reduction in insulin receptor content in the liver, and only in the liver (19). Since the Alb-Cre transgene is only expressed in hepatocytes, and since hepatocytes make up only about $85 \%$ of the total number of cells in the liver, it is likely that the knockout of insulin receptor in LIRKO hepatocytes is essentially complete. LIRKO mice have an absence of insulin-stimulated receptor tyrosine phosphorylation and insulin signaling in the hepatocyte (19). Since the livers of LIRKO mice cannot respond directly to insulin, any effect of insulin administered in vivo must be mediated by indirect actions of insulin on extrahepatic tissues.

In this study, in an attempt to clarify indirect effects of insulin on the suppression of HGP, we assessed the regulation of HGP in both control and LIRKO mice by performing high-dose hyperinsulinemic-euglycemic clamps to maximally stimulate insulin-sensitive peripheral tissues. We speculated that the marked chronic hyperinsulinemia in the LIRKO mice might have led to secondary insulin resistance in muscle and fat (20-22) and perhaps limited insulin action on these extrahepatic tissues. We therefore treated a second group of LIRKO mice with streptozotocin (LIRKO+STZ mice) to reduce hyperinsulinemia in an 
attempt to improve peripheral insulin sensitivity. Our goal was to maximize the potential to observe indirect actions of insulin to limiting gluconeogenic precursor supply and thus suppress HGP.

\section{Methods}

Animals. IR ${ }^{\text {Lox-Lox }}$ mice homozygous for the floxed insulin receptor allele were bred with transgenic mice that express the Cre recombinase cDNA from the rat albumin promoter to generate IR ${ }^{\text {Lox-Lox:alb-Cre }}{ }^{+/}$LIRKO mice (19, $23,24)$. Genotypes were determined by PCR of tail DNA (23). A group of 9-week-old LIRKO mice were rendered diabetic with an intraperitoneal injection of streptozotocin (STZ; $125 \mu \mathrm{g} / \mathrm{kg}$ daily for three days) and were given no insulin treatment during a 3 -week recovery period prior to experimentation. These diabetic LIRKO+STZ mice had a marked reduction in endogenous insulin levels. All mice were housed on a 12-hour light/dark cycle and fed a standard rodent chow ad libitum (Mouse Diet 9F; PMI Nutrition International, St. Louis, Missouri, USA; percent of calories from carbohydrates, $56.5 \%$; from fat, $21.6 \%$; from protein, $21.9 \%$ ). All procedures were conducted in accordance with NIH guidelines for the care and use of laboratory animals and were approved by the Joslin Diabetes Center Animal Care Committee.

Euglycemic-byperinsulinemic glucose clamps. Twelve-weekold mice were given an intraperitoneal injection of an anesthetic cocktail containing tert-amyl alcohol (SigmaAldrich, St. Louis, Missouri, USA) and tribromoethanol (Sigma-Aldrich). After loss of pedal and corneal reflexes was assured, a catheter (Micro-Renathane, MRE 025; Braintree Scientific Inc., Braintree, Massachusetts, USA) was inserted into the right internal jugular and advanced to the level of the superior vena cava. The catheter was filled with saline, tunneled, and left in a subcutaneous pocket at the back of the neck. A 3-0 silk suture was attached to the free end of the catheter and exteriorized in such a fashion as to allow retrieval of the catheter on the day of experimentation. Mice were given $1 \mathrm{ml}$ of subcutaneous saline and allowed to recover on a heating pad.

After 4-6 days of recovery, only mice that had lost less than $10 \%$ of their preoperative weight were studied. Each animal was fasted for 5 hours on the morning of the experiment and then placed in a restrainer to which it was accustomed. HPLC-purified [3-3 $\left.{ }^{3} \mathrm{H}\right]$-glucose tracer (NEN Life Science Products Inc., Boston, Massachusetts, USA) was infused intravenously $(10 \mu \mathrm{Ci}$ bolus, $0.1 \mu \mathrm{Ci} / \mathrm{min})$. After an 80-minute basal period, a blood sample was collected from the tail tip for determination of basal glucosespecific activity and plasma concentration of glucose, insulin, glucagon, and FFAs. To minimize blood loss, rbc's were collected by centrifugation, resuspended in saline, and reinfused. At time 0 , regular human insulin was infused (Novolin R; Novo Nordisk Pharmaceutical Industries Inc., Clayton, North Carolina, USA; 200 $\mathrm{mU} / \mathrm{kg}$ bolus, $20 \mathrm{mU} / \mathrm{kg} / \mathrm{min}$ ). The insulin infusion solution was prepared by dilution of insulin with saline containing 0.1\% BSA (Sigma-Aldrich). Blood samples $(2.2 \mu \mathrm{l})$ were subsequently drawn at 10-minute intervals for the determination of blood glucose (Glucometer Elite; Bayer Corp., Elkhart, Indiana, USA). An infusion of 50\% dextrose (Abbott Laboratories, Chicago, Illinois, USA) was adjusted to maintain plasma glucose between 100 and $125 \mathrm{mg} / \mathrm{dl}$. Steady state was ascertained when a fixed glucose infusion rate maintained blood glucose measurements constant for 30 minutes. This steady state was achieved within 90-120 minutes, at which time a blood sample was collected for determination of glucose radioactivity and plasma concentration of glucose, insulin, glucagon, and FFAs.

Assays. Insulin concentrations were determined by ELISA using mouse-insulin antibody (Crystal Chem Inc., Chicago, Illinois, USA). Glucagon was determined by RIA (Linco Research Inc., St. Charles, Missouri, USA). Plasma nonesterified fatty acids (FFAs) were measured by an enzymatic colorimetric method (Wako Chemicals USA Inc., Richmond, Virginia, USA). Plasma glucose was assayed by the glucose oxidase method (Glucose Analyzer; Beckman Instruments Inc., Palo Alto, California, USA). Plasma $\left[3-{ }^{3} \mathrm{H}\right]$-glucose radioactivity was measured after deproteinization with barium hydroxide and zinc sulphate, evaporation to removed tritiated water, and counting in a liquid scintillation counter (Beckman Instruments Inc.).

Calculations. Glucose turnover rate $(\mathrm{mg} / \mathrm{kg} / \mathrm{min})$ was calculated during the basal period and during the steady-state portion of the clamp as the rate of tracer infusion (disintegrations per minute/min) divided by the plasma glucose-specific activity (disintegrations per minute/mg) corrected to the body weight. During the clamp, HGP was calculated as the difference between the tracer-derived rate of glucose appearance and the infusion rate of glucose. Glucose turnover was equal to glucose utilization under all conditions except in the LIRKO-STZ mice during the hyperglycemic basal period, when glycosuria likely contributed to total glucose turnover. All results are presented as the mean \pm SEM. Statistical significance $-P<0.05$ unless otherwise noted - was determined by Student's $t$ test.

\section{Results}

Basal metabolic parameters. Fasting blood glucose was higher in the Lox-Lox control group than in LIRKO mice (112 \pm 6 vs. $88 \pm 6 \mathrm{mg} / \mathrm{dl}$ ) (Table 1; Figure 1a). Basal insulin levels were elevated almost tenfold in LIRKO mice $(4,907 \pm 1,212 \mathrm{pg} / \mathrm{ml})$ as compared with Lox-Lox control mice $(497 \pm 116 \mathrm{pg} / \mathrm{ml}$ ) (Table 1; Figure 2a). STZ treatment significantly lowered basal insulin levels by $60 \%$ in LIRKO + STZ mice $(1,916 \pm 694 \mathrm{pg} / \mathrm{ml})$, although these levels were still elevated as compared with those in Lox-Lox control mice $(P<0.05)$ (Table 1; Figure 2a). With the reduction of hyperinsulinemia, LIRKO $+\mathrm{STZ}$ mice had threefold higher glucose levels $(335 \pm 40 \mathrm{mg} / \mathrm{dl}$, $P<0.01$ ) (Table 1; Figure 1a), which indicates the important role of increased insulin action on tissues such as muscle and fat to compensate for hepatic insulin resistance and allow near normal blood glucose in the LIRKO mouse. Basal FFA levels were similar in the Lox-Lox 


\section{Table 1}

Basal characteristics of control Lox-Lox, LIRKO, and LIRKO mice made diabetic with STZ treatment

\begin{tabular}{|c|c|c|c|}
\hline Fasting & $\begin{array}{l}\text { Lox-Lox } \\
(n=6)\end{array}$ & $\begin{array}{l}\text { LIRKO } \\
(n=7)\end{array}$ & $\begin{array}{l}\text { LIRKO+STZ } \\
\quad(n=6)\end{array}$ \\
\hline Glucose (mg/dl) & $112 \pm 6$ & $88 \pm 6^{A}$ & $335 \pm 40^{\mathrm{B}, \mathrm{D}}$ \\
\hline Insulin ( $\mathrm{pg} / \mathrm{ml})$ & $497 \pm 116$ & $4,907 \pm 1,212^{\mathrm{B}}$ & $1,916 \pm 694^{\mathrm{A}, \mathrm{C}}$ \\
\hline FFAs $(\mathrm{mEq} / \mathrm{l})$ & $0.854 \pm 0.112$ & $0.823 \pm 0.119$ & $1.445 \pm 0.240^{A, C}$ \\
\hline
\end{tabular}

$(0.854 \pm 0.112 \mathrm{mEq} / \mathrm{l})$ and LIRKO $(0.823 \pm 0.119 \mathrm{mEq} / \mathrm{l}$, $P=$ not significant $[\mathrm{NS}])$ groups but were significantly elevated in the diabetic LIRKO+STZ group $(1.445 \pm 0.240$ $\mathrm{mEq} / \mathrm{l})$ (Table 1; Figure 2b). Basal glucagon levels were similar in the Lox-Lox and LIRKO groups (60 \pm 12 and $47 \pm 9 \mathrm{pg} / \mathrm{ml}$, respectively, $P=\mathrm{NS})$ but were elevated in the LIRKO + STZ group $(122 \pm 31 \mathrm{pg} / \mathrm{ml}, P<0.05)$ (Figure 2c). Somewhat surprisingly, basal HGP as assessed with $\left[3-{ }^{3} \mathrm{H}\right]$-glucose tracer was similar in all three groups (Lox-Lox $22.0 \pm 2.8$, LIRKO $18.9 \pm 1.8$, and LIRKO+STZ $22.9 \pm 6.3 \mathrm{mg} / \mathrm{kg} / \mathrm{min}$ ) (Figure 3a).

Hyperinsulinemic-englycemic clamp. By experimental design, the high dose of insulin infusion during the clamp markedly raised insulin levels, and to a similar extent in all groups (Lox-Lox 17,632 $\pm 2,975$, LIRKO $21,067 \pm 5,276$, and LIRKO+STZ 24,435 $\pm 6087 \mathrm{pg} / \mathrm{ml}$, $P=$ NS) (Figure 2a). Blood glucose was effectively clamped at euglycemic levels and was not significantly different between the groups (Lox-Lox $109 \pm 10$, LIRKO $115 \pm 12$, and LIRKO+STZ $102 \pm 6 \mathrm{mg} / \mathrm{dl}, P=\mathrm{NS}$ ) (Figure 1a). During the clamp, insulin suppressed FFA levels to a similar extent in all groups (Lox-Lox $0.500 \pm 0.077$, LIKRO $0.537 \pm 0.075$, and LIRKO+STZ $0.704 \pm 0.124$ $\mathrm{mEq} / \mathrm{l}$ ) (Figure $2 \mathrm{~b}$ ). Glucagon levels were not suppressed during the clamp in any group (Figure 2c).

In response to insulin, HGP was suppressed in the Lox-Lox group by $82 \% \pm 17 \%$ to $4.4 \pm 4.4 \mathrm{mg} / \mathrm{kg} / \mathrm{min}$ (Figure 3a). By contrast, HGP did not change significantly in response to insulin during the clamp in LIRKO $(22.3 \pm 2.5 \mathrm{mg} / \mathrm{kg} / \mathrm{min})$ and LIRKO+STZ mice $(17.7 \pm 8.0 \mathrm{mg} / \mathrm{kg} / \mathrm{min}, P=\mathrm{NS}$ for LIRKO vs. LIRKO+STZ). The failure to suppress HGP in LIRKO and LIRKO+STZ mice indicates profound hepatic insulin resistance.

During the clamp, insulin-stimulated glucose utilization was significantly impaired in LIRKO mice $(57.4 \pm 8.6$ $\mathrm{mg} / \mathrm{kg} / \mathrm{min}$ ) as compared with Lox-Lox controls (111.0 \pm $6.9 \mathrm{mg} / \mathrm{kg} / \mathrm{min}$ ) (Figure 3b). Tracer-independent glucose infusion rates were similarly impaired in LIRKO mice as compared with Lox-Lox controls (35.1 \pm 7.4 vs. $106.6 \pm 3.3$ $\mathrm{mg} / \mathrm{kg} / \mathrm{min}$ ), indicating profound insulin resistance (Figure 1b). Lowering chronic hyperinsulinemia with STZ treatment (LIRKO+STZ) improved clamp values for glucose utilization $(71.9 \pm 10.9 \mathrm{mg} / \mathrm{kg} / \mathrm{min})$ and glucose infusion $(54.2 \pm 5.7 \mathrm{mg} / \mathrm{kg} / \mathrm{min}, P=0.07$ vs. LIRKO), but these values failed to reach a statistically significant difference compared with those in the LIRKO group.

\section{Discussion}

In the absence of direct insulin signaling in the hepatocyte, LIRKO mice provide a unique model to determine the direct versus indirect effects of insulin on the suppression of HGP. The primary finding in this study is that high-dose insulin, chosen to maximize extrahepatic insulin action, failed to suppress glucose production; this suggests the absence of an indirect HGP-suppressing effect of insulin in this model. We also present evidence that the chronic hyperinsulinemia of the LIRKO mouse may induce secondary peripheral insulin resistance that can be reversed, in part, by lowering insulin levels with STZ. However, even when the chronic high insulin levels are lowered with STZ to improve peripheral insulin resistance and maximize the potential to observe indirect action on the liver, insulin again fails to acutely suppress HGP in STZ-treated LIRKO mice. These results indicate the critical importance of an intact insulin-signaling system in the liver that is necessary to reveal both direct and indirect actions of insulin in the suppression of HGP.

In the absence of hepatic insulin effects, LIRKO livers were previously noted to have decreased glycogen stores, increased expression of phosphoenolpyruvate carboxykinase and glucose-6-phosphatase, and decreased expression of glucokinase, all of which should favor increased rates of gluconeogenesis (19). It could be argued, since LIRKO livers are half the size of controls, that if HGP is normalized to liver mass, then

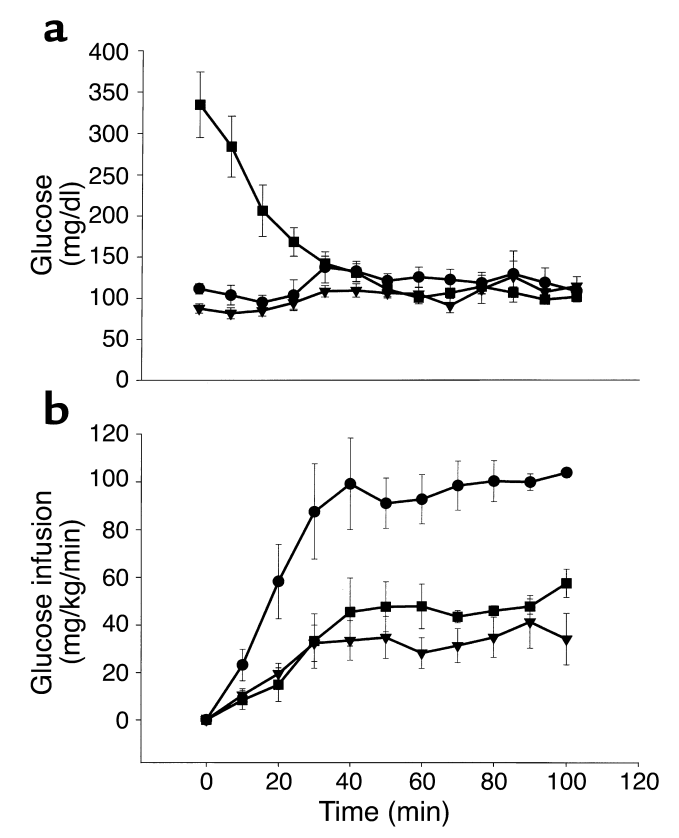

Figure 1

Blood glucose levels (a) and glucose infusion rates (b) during the euglycemic-hyperinsulinemic clamp. After basal sampling at time 0 , insulin was infused $(20 \mathrm{mU} / \mathrm{kg} / \mathrm{min})$ for the duration of the 2-hour clamps. Blood glucose (from tail-vein samples) was clamped at euglycemic levels $(\sim 125 \mathrm{mg} / \mathrm{dl})$ with intravenous infusion of dextrose (50\%). Treatment groups were Lox-Lox control, $n=6$ (circles); LIRKO, $n=7$ (triangles); and LIRKO+STZ, $n=6$ (squares). 


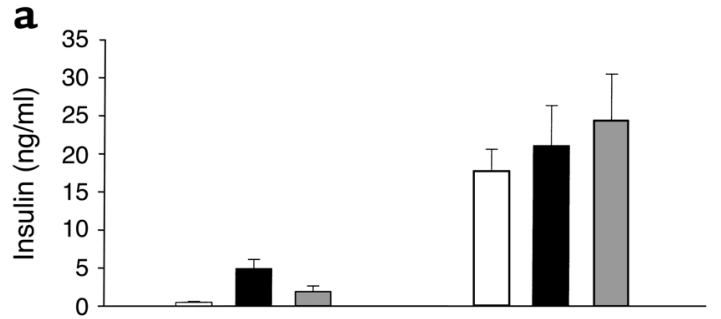

b
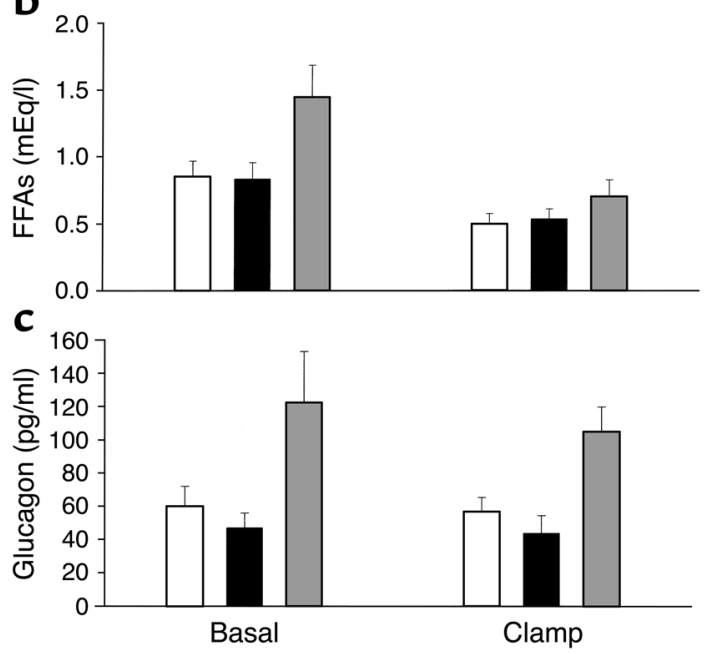

the LIRKO groups in this study have approximately twofold elevated rates of HGP, suggesting markedly increased gluconeogenesis per unit liver mass. Increased reliance on gluconeogenesis in LIRKO mice would be expected to potentiate indirect insulin action, but this was not observed during the clamp. Paradoxically, basal rates of HGP were similar in all three experimental groups despite marked differences in basal levels of hormones and metabolic substrates. One could hypothesize that the marked hyperinsulinemia of the LIRKO mouse plays a role in limiting excessive HGP in the basal state, but our study, noting the absence of an insulin effect on HGP, would argue against that hypothesis. Additionally, the fact that the reduction of chronic hyperinsulinemia with STZ treatment did not increase basal HGP supports our finding, during the clamp, of a lack of evidence for an indirect role of insulin in the suppression of glucose production.

Since most of the glucose disposal during a hyperinsulinemic clamp represents uptake into skeletal muscle, the markedly impaired glucose utilization in

\section{Figure 3}

HGP (a) and glucose turnover rates (b) in the basal period and in response to insulin infusion $(20 \mathrm{mU} / \mathrm{kg} / \mathrm{min})$ during the hyperinsulinemic clamp as assessed using $\left[3-{ }^{3} \mathrm{H}\right]$-glucose tracer dilution methodology. Surprisingly, in spite of varied insulin levels, there was no difference in basal HGP. During the hyperinsulinemic-euglycemic clamp, insulin suppressed HGP by $82 \% \pm 17 \%$ in control mice but failed to suppress HGP in both LIRKO and LIRKO+STZ mice. Data are shown for Lox-Lox controls, $n=6$ (white bars); LIRKO mice, $n=7$ (black bars); and LIRKO+STZ mice, $n=6$ (gray bars). Statistical differences between study groups are discussed in Results.

\section{Figure 2}

Insulin (a), FFA (b), and glucagon (c) levels in the basal period and in response to insulin infusion $(20 \mathrm{mU} / \mathrm{kg} / \mathrm{min})$ during the hyperinsulinemic clamp. Data are shown for Lox-Lox controls, $n=6$ (white bars); LIRKO mice, $n=7$ (black bars); and LIRKO+STZ mice, $n=6$ (gray bars). Statistical differences between study groups are discussed in Results.

LIRKO mice, as compared with control mice, reflects profound skeletal-muscle insulin resistance. Interestingly, this extrahepatic insulin resistance may be specific for muscle, based on the following observations. Normal basal FFA levels in LIRKO mice suggest normal whole-body rates of lipid turnover, and the similar suppression of FFA levels in response to insulin suggests normal insulin sensitivity with respect to antilipolytic actions on adipocytes in LIRKO mice. Therefore, in addition to primary tissue-specific (hepatic) insulin resistance in LIRKO mice, the secondary insulin resistance caused in part by hyperinsulinemia may also be tissue-selective, with a much greater effect in muscle than in fat.

The extrahepatic, i.e., secondary, insulin resistance in the LIRKO mouse may be mediated by chronic hyperinsulinemia. STZ treatment of LIRKO mice reduced hyperinsulinemia and tended to improve glucose utilization (e.g., extrahepatic insulin sensitivity), although this improvement failed to reach statistical significance. We speculate that chronic hyperglycemia, hyperlipidemia, and residual hyperinsulinemia in the LIRKO+STZ group may have masked the trend toward improvement of extrahepatic insulin sensitivity. Interestingly, the known toxic metabolic effects of high glucose and FFA levels failed to worsen insulin sensitivity

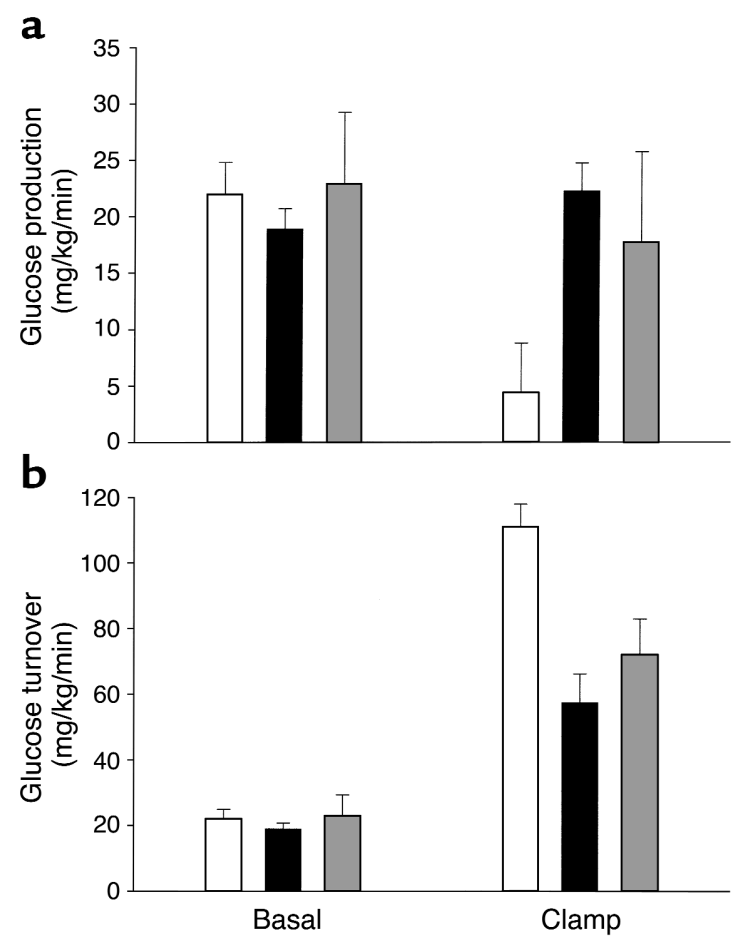


in the LIRKO+STZ mice. These results suggest that in this model, chronic hyperinsulinemia is a more important contributor to peripheral insulin resistance than are chronic hyperglycemia and hyperlipidemia.

Endogenous glucose production in this study was determined by dilution of $\left[3-{ }^{3} \mathrm{H}\right]$-glucose in the basal and clamp conditions. Under normal circumstances in humans, most endogenous glucose production is derived from the liver, with renal glucose release contributing only $5-20 \%(25,26)$. The liver and kidneys both suppress glucose release in response to insulin. Although individual tissue glucose production, e.g., contribution of liver versus kidney, has not been determined in mouse models, in our study we would have expected to observe a significant insulin-induced suppression of glucose release in the LIRKO and $\mathrm{LIRKO}+\mathrm{STZ}$ mice if endogenous glucose production included a large contribution by kidney or other extrahepatic sources. The absence of a suppression of glucose production in this study suggests that the extrahepatic contribution to calculated endogenous glucose production was minimal.

The role of glucagon in mediating the indirect effects of insulin on HGP is controversial. Suppression of glucagon has been implicated as an important indirect mediator of the insulin-induced suppression of $\operatorname{HGP}(2$, $17,18)$, although the indirect effect of insulin via suppression of substrates may still exist under conditions of maintained glucagon levels $(1,3,4)$. Since elevated glucagon levels may potentiate the direct effect of insulin on the liver, we previously hypothesized that the main direct effect of insulin on the liver is to counteract the effect of glucagon $(3,17,18)$. In the current study, it is possible that the failure of insulin to suppress glucagon secretion in the LIRKO group is related to $\alpha$ cell insulin resistance. The increase in basal glucagon levels upon removal of chronic hyperinsulinemia with STZ treatment supports this hypothesis. However, under the conditions of our study, acute insulin administration failed to suppress glucagon in any group.

High glucagon levels did not result in an increase in basal HGP in the LIRKO+STZ mice, although, arguably, such an increase may have been masked by concurrent hyperglycemia, which is known to limit HGP. Our data indicate two distinct lines of evidence suggesting a minimal involvement of glucagon as a regulator of HGP. First, the failure of hyperglucagonemia to increase basal HGP in the LIRKO+STZ mice, and second, the observation that insulin suppressed HGP in the control group without changing glucagon levels, argue against a significant role for glucagon. Clearly the regulation of glucagon secretion and the effect of glucagon on HGP in the rodent may be complex and will require further characterization.

Elevated FFA levels contribute to insulin resistance in the liver and to increased rates of gluconeogenesis in studies of people with and without diabetes (27-32). Classically, hepatic FFA oxidation promotes gluconeogenesis via production of ATP, NADH, and
acetyl-CoA (to activate pyruvate carboxylase) (33). Suppression of hepatic FFA flux, and presumably hepatic FFA oxidation, has consistently been shown to be the dominant mechanism by which actions of insulin in the peripheral circulation can indirectly suppress HGP (7-10). As an alternative mechanism of action, FFAs can contribute to insulin resistance via modulation of insulin signaling. FFA infusion causes a reduction in insulin-stimulated insulin receptor substrate-1-associated (IRS-1-associated) PI3K activity $(34,35)$. Similarly, increased hepatic intracellular fatty acid-derived metabolites result in defects in insulin activation of IRS-2-associated PI3K activity and an impaired ability of insulin to suppress endogenous glucose production in transgenic mice (36) and in rats fed high-fat diets (37). Our findings that insulin suppressed FFAs but failed to suppress glucose production in LIRKO mice demonstrate an absence of an indirect effect of reduced FFA flux in mediating hepatic glucose turnover. If FFA-substrate flux to the liver is indeed a dominant extrahepatic regulator of HGP, our results suggest that this metabolic cross-talk requires the presence of an intact insulinsignaling network. Our findings support a unifying hypothesis that FFA-mediated insulin-induced suppression of HGP occurs via direct modulation of insulin signaling in the liver.

It might be argued that the chronic lack of hepatic insulin action in the LIRKO mouse may lead to a loss of the normal ability of the hepatic glucose production to be regulated by substrate supply. However, in previous work in depancreatized dogs, one of us (S.J. Fisher) has shown that the inhibition of FFA oxidation suppressed HGP by $25 \%$ (38). These findings demonstrate that the integrity of a substrate-mediated suppression of HGP exists in the setting of complete insulin deficiency but an intact insulin-signaling network. These observations are consistent with our current findings, which suggest that the substrate-mediated indirect suppression of HGP may be mediated by interaction with the insulin-signaling network in the liver.

We conclude that in LIRKO mice, (a) insulin, even at high concentrations, fails to suppress glucose production, indicating that both the direct and the indirect effects of insulin require an intact insulin-signaling pathway in the liver; (b) the marked hyperinsulinemia associated with primary hepatic insulin resistance leads to secondary extrahepatic insulin resistance; and (c) lowering insulin levels with STZ produces hyperglycemia and hyperlipidemia, but the improvement in extrahepatic insulin sensitivity fails to reveal the previously postulated indirect role of insulin in the suppression of glucose production.

\section{Acknowledgments}

We thank S. Lannon, S.E. Curtis, R. Quinn, and E. Fletcher for providing valuable technical assistance, and M.D. Michael and P.G. Miles for valuable discussions. This work was supported by NIH grant R01 DK31036. 
1. Ader, M., and Bergman, R.N. 1990. Peripheral effects of insulin dominate suppression of fasting hepatic glucose production. Am. J. Physiol. 245:E1020-E1032.

2. Giacca, A., et al. 1992. Importance of peripheral insulin levels for insulin-induced suppression of glucose production in depancreatized dogs. J. Clin. Invest. 90:1769-1777.

3. Mittelman, S.D., Fu, Y.Y., Rebrin, K., Steil, G.M., and Bergman, R.N. 1997. Indirect effect of insulin to suppress endogeneous glucose production is dominant, even with hyperglucoagonemia. J. Clin. Invest. 100:3121-3130.

4. Sindelar, D.K., Balcom, J.H., Chu, C.A., Neal, D.W., and Cherrington, A.D. 1996. A comparison of the effects of selective increases in peripheral or portal insulin on hepatic glucose production in the conscious dog. Diabetes. 45:1594-1605.

5. Lewis, G.F., Zinman, B., Groenwoud, Y., Vranic, M., and Giacca, A. 1996 Hepatic glucose production is regulated both by direct hepatic and extra hepatic effects of insulin in humans. Diabetes. 45:454-462.

6. Lewis, G.F., Carpentier, A., Vranic, M., and Giacca, A. 1999. Resistance to insulin's acute direct hepatic effect in suppressing steady-state glucose production in individuals with type 2 diabetes. Diabetes. 48:570-576

7. Rebrin, K., Steil, G.M., Getty, L., and Bergman, R.N. 1995. Free fatty acid as a link in the regulation of hepatic glucose output by peripheral insulin. Diabetes. 44:1038-1045.

8. Lewis, G.F., Vranic, M., Harley, P., and Giacca, A. 1997. Fatty acids mediate the acute extrahepatic effects of insulin on hepatic glucose production in humans. Diabetes. 46:1111-1119.

9. Sindelar, D., Chu, C., Rohlie, M., Neal, D.W., and Swift, L.L. 1997. The role of fatty acids in mediating the effects of peripheral insulin on hepatic glucose production in the conscious dog. Diabetes. 46:187-196.

10. Mittelman, S.D., and Bergman, R.N. 2000. Inhibition of lipolysis causes suppression of endogenous glucose production independent of changes in insulin. Am. J. Physiol. Endocrinol. Metab. 279:E630-E637.

11. Consoli, A., Nurjhan, N., Capani, F., and Gerich, J. 1989. Predominant role of gluconeogenesis in increased hepatic glucose production in NIDDM. Diabetes. 38:550-557.

12. Magnusson, I., Rothman, D.L., Katz, L.D., Shulman, R.G., and Shulman, G.I. 1992. Increased rate of gluconeogenesis in type II diabetes mellitus. A $13 \mathrm{C}$ nuclear magnetic resonance study. J. Clin. Invest. 90:1323-1327.

13. Hwang, J.H., et al. 1995. Impaired net hepatic glycogen synthesis in insulin-dependent diabetic subjects during mixed meal ingestion: $\mathrm{a}^{13} \mathrm{C}$ nuclear magnetic resonance spectroscopy study. J. Clin. Invest. 95:783-787.

14. Boden, G., Chen, X., and Stein, T.P. 2001. Gluconeogenesis in moderately and severely hyperglycemic patients with type 2 diabetes mellitus. Am. J. Physiol. Endocrinol. Metab. 280:E23-E30.

15. Gupta, N., et al. 1998. Euglycemia acutely restores the direct hepatic effect of insulin on glucose production in depancreatised dogs. Diabetes. 44:A41. (Abstr.)

16. Stevenson, R.W., Williams, P.E., and Cherrington, A.D. 1987. Role of glucagon suppression on gluconeogenesis during insulin treatment of the conscious diabetic dog. Diabetologia. 30:782-790.

17. Giacca, A., Fisher, S.J., McCall, R.H., Shi, Z.Q., and Vranic, M. 1997. Direct and indirect effects of insulin in suppressing glucose production in depancreatized dogs: role of glucagon. Endocrinology. 138:999-1007.

18. Lewis, G.F., Vranic, M., and Giacca, A. 1997. Glucagon enhances the direct suppressive effect of insulin on hepatic glucose production in humans. Am. J. Physiol. 272:E371-E378.
19. Michael, M.D., et al. 2000. Loss of insulin signaling in hepatocytes leads to severe insulin resistance and progressive hepatic dysfunction. Mol. Cell. 6:87-97.

20. Rizza, R.A., Mandarino, L.J., Genest, J., Baker, B.A., and Gerich, J.E. 1985. Production of insulin resistance by hyperinsulinaemia in man. Diabetologia. 28:70-75.

21. Marangou, A.G., et al. 1986. Metabolic consequences of prolonged hyperinsulinemia in humans. Evidence for induction of insulin insensitivity. Diabetes. 35:1383-1389.

22. Radziuk, J., Barron, P., Najm, H., and Davies, J. 1993. The effect of systemic venous drainage of the pancreas on insulin sensitivity in dogs. J. Clin. Invest. 92:1713-1721.

23. Bruning, J.C., et al. 1998. A muscle-specific insulin receptor knockout exhibits features of the metabolic syndrome of NIDDM without altering glucose tolerance. Mol. Cell. 2:559-569.

24. Postic, C., et al. 1999. Dual roles for glucokinase in glucose homeostasis as determined by liver and pancreatic $\beta$ cell-specific gene knock-outs using Cre recombinase. J. Biol. Chem. 274:305-315.

25. Gerich, J.E., Meyer, C., Woerle, H.J., and Stumvoll, M. 2001. Renal gluconeogenesis: its importance in human glucose homeostasis. Diabetes Care. 24:382-391.

26. Moller, N., Rizza, R.A., Ford, G.C., and Nair, K.S. 2001. Assessment of postabsorptive renal glucose metabolism in humans with multiple glucose tracers. Diabetes. 50:747-751.

27. Bevilacqua, S., et al. 1990. Operation of Randle's cycle in patients with NIDDM. Diabetes. 39:383-389.

28. Fanelli, C., et al. 1993. Demonstration of a critical role for free fatty acids in mediating counterregulatory stimulation of gluconeogenesis and suppression of glucose utilization in humans. J. Clin. Invest. 92:1617-1622.

29. Saloranta, C., et al. 1993. Contribution of muscle and liver to glucosefatty acid cycle in humans. Am. J. Physiol. 264:E599-E605.

30. Boden, G., Chen, X., Ruiz, J., White, J.V., and Rossetti, L. 1994. Mechanisms of fatty acid-induced inhibition of glucose uptake. J. Clin. Invest. 93:2438-2446

31. Chu, C.A., et al. 2002. Effects of free fatty acids on hepatic glycogenolysis and gluconeogenesis in conscious dogs. Am. J. Physiol. Endocrinol. Metab. 282:E402-E411.

32. Boden, G., Chen, X., Capulong, E., and Mozzoli, M. 2001. Effects of free fatty acids on gluconeogenesis and autoregulation of glucose production in type 2 diabetes. Diabetes. 50:810-816

33. Williamson, J.R., Kreisberg, R.A., and Felts, P.W. 1966. Mechanism for the stimulation of gluconeogenesis by fatty acids in perfused rat liver. Proc. Natl. Acad. Sci. USA. 56:247-254.

34. Griffin, M.E., et al. 1999. Free fatty acid-induced insulin resistance is associated with activation of protein kinase $\mathrm{C}$ theta and alterations in the insulin signaling cascade. Diabetes. 48:1270-1274.

35. Dresner, A., et al. 1999. Effects of free fatty acids on glucose transport and IRS-1-associated phosphatidylinositol 3-kinase activity. J. Clin. Invest. 103:253-259.

36. Kim, J.K., et al. 2001. Tissue-specific overexpression of lipoprotein lipase causes tissue-specific insulin resistance. Proc. Natl. Acad. Sci. USA. 98:7522-7527.

37. Samuel, V.T., Yu, C., Chen, Y., Zong, H., and Shulman, G.I. 2002. Mechanism of fat induced hepatic insulin resistance. Diabetes. 51(Suppl. 2):A68. (Abstr.)

38. Yamatani, K., et al. 1992. Role of FFA-glucose cycle in glucoregulation during exercise in total absence of insulin. Am. J. Physiol. 263:E646-E653. 